\title{
Dynamic settling of particles in shear flows of shear-thinning fluids
}

\author{
L.H. Childs ${ }^{\mathrm{a}}$, A.J. Hogg ${ }^{\mathrm{a}, *}$, D. Pritchard ${ }^{\mathrm{b}}$ \\ ${ }^{a}$ School of Mathematics, University of Bristol, University Walk, Bristol BS8 1TW, U.K. \\ ${ }^{b}$ Dept of Mathematics and Statistics, University of Strathclyde, 26 Richmond Street, \\ Glasgow G1 1XH, Scotland, U.K.
}

\begin{abstract}
Dynamic settling is the phenomenon whereby a relatively dense particle settles through a sheared flow of a non-Newtonian fluid at a speed that depends on the shear rate of the background flow. This means that due to the nonlinear rheology, the settling velocity may vary spatially and temporally as the background shear rate of the suspending fluid varies, an effect which does not occur in Newtonian fluids. In this contribution, the consequences of this dependency are explored for a dilute suspension of particles released uniformly from a source in a sustained and externally-driven flow of shear-thinning fluid. It is shown theoretically that the concentration field does not remain uniform, but evolves downstream, allowing calculation of the runout length, settling times and distribution of the deposited particles. Flows with a velocity maximum are demonstrated to affect the concentration field very strongly as they develop a 'kinematic barrier' over which settling times are considerably lengthened. Flows with bidisperse suspensions are shown to produce deposits that vary nonmonotonically in thickness and composition with distance downstream, an effect which is solely due to dynamic settling. Finally flows of viscoplastic fluids which exhibit yielded and unyielded regions may accentuate the role and effects of the kinematic barrier to settling.
\end{abstract}

\section{Introduction}

The transport of relatively dense particles in a flow of a shear-thinning or viscoplastic fluid occurs in many natural and industrial settings. Examples include the transport of cuttings by drilling mud [1], proppant emplacement in hydraulic fracturing (e.g. [2], §6.2), the transport of coarse material such as sand and gravel in a fine-grained slurry $[3,4]$ or debris flow $[5,6]$ and the handling of two-phase materials in food processing [7]. Key questions for modelling such flows include the distance that particles can be transported before settling out,

\footnotetext{
${ }^{*}$ Corresponding author

Email address: a.j.hogg@bris.ac.uk (A.J. Hogg)
} 
the time they take to do so, the geometry of the deposit, and the distribution of grain sizes within it.

A small particle suspended in an otherwise quiescent non-Newtonian fluid will typically settle in a laminar regime at a constant speed $[8,9,10]$, unless the gravitational stress that the dense particle exerts is unable to overcome the yield stress of the suspending fluid [11]. However, if the fluid is not quiescent then the nonlinearity of the rheology means that the background flow affects the settling. In a flow which is sheared on a rather larger scale than the scale of the particle, the background shear rate determines the local viscosity of the fluid, which in turn determines the settling velocity. An extreme case occurs when the fluid has a yield stress, in which case small suspended particles may be supported indefinitely when the fluid is static, but then settle when the fluid is set in motion by forces that exceed the yield stress.

This dependence of settling velocity on the background flow is sometimes referred to as 'dynamic settling' and has been known for many years in the oil and process engineering communities $[12,13,2,14,15]$. Because it allows particles to be carried long distances in relatively low-shear flows, it can be a desirable effect in, for example, the transport of drill cuttings; however, it is among the factors implicated in undesirable phenomena such as barite sag [16]. (Confusingly, it may act in conjunction with Boycott settling, also referred to in the literature as 'dynamic settling'.) Under the name of 'competence variation' [5], it has also been proposed as a mechanism for inverse grading in deposits from muddy flows.

Despite these applications, dynamic settling has hitherto received relatively little attention from fluid dynamicists. Notable experimental contributions have included those of Merkak et al. [17, 18], who studied experimentally the flow and sedimentation of small particles suspended in a viscoplastic gel, and that of Ovarlez et al. [19] has examined experimentally the shear-induced sedimentation of relatively small particles in yield stress fluids using MRI to determine the evolving concentration within a sheared Couette device. The most substantial modelling contribution has been that of Angelis and Mancini [20] (and see also the review by [4]), who used an empirical settling velocity correlation to calculate the trajectories of particles settling within a viscoplastic pipe flow and feeding a mobile dense layer at the bottom of the pipe, under which a static deposit grew.

In this contribution, we analyse a simple model of dynamic settling in horizontal shear flows of a shear-thinning fluid, and investigate the fate of particles as they are transported downstream and settle out of suspension. This model reveals that dynamic settling may have a number of interesting consequences, including strong effects on transport distances, the development of statically unstable particle concentration gradients within the flow, and the formation of deposits that neither thin nor become finer monotonically with distance downstream from the source. The latter two effects cannot occur with particles settling from a flowing Newtonian fluid, for which the settling velocity is constant and increases monotonically with particle size. Instead the development from a uniform source of statically unstable concentration gradients and spa- 
tially varying deposits is due to dynamic settling. The vital coupling in shear flows of shear-thinning fluids that leads to these phenomena is that the settling velocity is reduced in regions of low shear rates and thus these zones can act as 'kinematic barriers' to settling particles.

The situation is more complicated when the suspending fluid possesses a yield stress, because relatively heavy particles could be fully supported within unyielded regions and even if they are of a sufficient submerged weight to overcome the yield stress, their sedimentation is affected by the yield stress [11]. In this study, we examine theoretically how a yield stress, and associated unyielded regions within a flow with spatially varying shear rates, influence dynamic settling. We focus on particles that are not arrested within the unyielded regions (unlike [20]) and we examine how the yield-stress effects complement the kinematic barrier due to the shear-thinning properties of the fluid.

We formulate a model for the flow in $\S 2$, basing our exposition on powerlaw fluids. We show in the appendix that an equivalent analysis can be carried out for fully developed flows of any generalised Newtonian fluid, but for the purposes of discussing the interplay of dynamical processes in these flows, we employ the simple power-law rheology (and later when analysing viscoplastic flows, the Herschel-Bulkley rheology). We tackle theoretically three related problems, which illustrate the consequences of dynamic settling. First we analyse settling within a horizontal free-surface flow driven by a constant pressure gradient (§3). We show how a non-uniform distribution of concentration arises due to dynamic settling effects. We then analyse the suspension within a twodimensional channel, also driven by a constant pressure gradient $(\S 4)$. The imposition of a no-slip condition at the upper surface retards the fluid motion and introduces an interior velocity maximum. We show that the maximum distance propagated by the particles within the channel flow is always less than in a free-surface flow of the same depth, driven by the same pressure gradient, but that the time taken for full settling to occur is always increased. However, somewhat counter-intuitively, for strongly shear-thinning fluids, the median of the depositional flux can be further from source in the channel flow than in the free-surface flow. We analyse a dilute bidisperse suspension ( $\$ 5)$ and show that dynamic settling alone can lead to compositional variations within the deposit. Finally in $\S 6$ we demonstrate the effects of a yield stress on settling through a horizontal channel flow, focusing on the role of the unyielded plug at the centre of the channel.

\section{Formulation}

We study the sedimentation of dilute suspensions of relatively dense particles within a shear flow of a non-Newtonian fluid, the motion of which is driven by an imposed horizontal pressure gradient. The particles are transported by the flowing interstitial fluid, but due to their excess density settle under the action of gravity and form a deposit on the underlying boundary.

We analyse the motion of the fluid and suspension in two spatial dimensions, with the coordinate axes aligned such that the $x$ axis is horizontal and 
streamwise, while the $z$ axis is vertical; unit vectors aligned with the $x$ and $z$ axes are denoted by $\hat{\mathbf{x}}$ and $\hat{\mathbf{z}}$, respectively. The fluid motion is steady and fully developed so that the velocity field is given by $\mathbf{u}=u(z) \hat{\mathbf{x}}$. It is driven by a sustained pressure gradient, $-G \hat{\mathbf{x}}$. The deviatoric shear stress is denoted by $\tau_{x z}$ and momentum balance leads to

$$
\frac{\partial \tau_{x z}}{\partial z}=-G
$$

The concentration of particles suspended in the fluid is denoted by $C(x, z, t)$ and the equation governing its evolution is

$$
\frac{\partial C}{\partial t}+\nabla \cdot\left(\mathbf{u}_{p} C\right)=0
$$

where the velocity field with which the concentration is advected is denoted $\mathbf{u}_{p}$. The diffusivity of the solid phase has been neglected because the particles are assumed to be sufficiently large that they are not affected by molecular fluctuations within the fluid. The suspension is dilute so that there are negligible interactions between the individual particles, and the inertia of the particles is also assumed negligible, so that the drag and gravitational forces that act upon them are in balance. Thus we write the instantaneous relationship between the velocity of an individual particle, the velocity of the fluid and gravitational settling

$$
\mathbf{u}_{p}=u \hat{\mathbf{x}}-w_{s} \hat{\mathbf{z}}
$$

In contrast to settling through fluid of Newtonian rheology, the settling velocity of the particles depends upon the motion of the interstitial fluid and the consequences of this dependence will be explored below. The particles settle out of the flow to the underlying boundary and build up a deposit of thickness $\eta(x, t)$. Its growth is determined by the settling flux at the boundary and is given by

$$
\left(1-\phi_{b}\right) \frac{\partial \eta}{\partial t}=w_{s} C(x, \eta, t)
$$

where $\phi_{b}$ is the volume fraction of particles within the deposit.

In this study we calculate the unsteady development of the suspension and the deposit due to a sustained source of particles imposed at $x=0$ and initiated at $t=0$. We thus impose that $C(0, z, t)=C_{0}$ and that initially the flow is otherwise free of particles, $C(x, z, 0)=0$. The deposit is also initially of vanishing thickness $(\eta(x, 0)=0)$. Since the flows are dilute, the growth of this deposit does not significantly alter the geometry of the boundary unless the motion is sustained for a long duration, and consequently it does not feed back upon the motion of the fluid phase. Expressed dimensionally this criterion requires that the deposit depth is much less than the flow depth $(\eta \ll h)$, which in turn demands that the duration of the flow must be much less than $h\left(1-\phi_{b}\right) /\left(w_{s} C\right)$.

To progress we adopt a particular rheology; the analysis could be performed rather generally (see Appendix A), but for the simplest exposition of the ideas, 
we focus on a power-law rheology, which encompasses the key feature of shear thinning - and it is this property that plays a vital role in what follows. We therefore assume that the interstitial fluid is of power-law rheology with flow index $n$ and consistency $K_{n}$, which is extended to include a yield stress in $\S 6$. The fully developed flow is then governed by

$$
\frac{\partial}{\partial z}\left(K_{n}\left|\frac{\partial u}{\partial z}\right|^{n-1} \frac{\partial u}{\partial z}\right)=-G .
$$

The model of the settling velocity plays an important role in what follows and leads to different behaviour from the Newtonian counterpart. Here we argue that settling is determined by a balance between the gravitational forces and the 'viscous' drag on the particle. Thus it must be inversely proportional to the 'local' viscosity of the fluid. For a power-law fluid, the effective local viscosity in a simple shear flow is $K_{n}|\partial u / \partial z|^{n-1}$ and then the settling velocity is inversely proportional to $|\partial u / \partial z|^{n-1}$ (see Ovarlez et al. [19]). Crucially it is dependent upon the shear rate of the suspending fluid provided $n \neq 1$ (i.e. provided the fluid is non-Newtonian). The settling velocity of an individual spherical particle in an imposed linear shear flow has been computed by Childs [21]. When the particle is sufficiently small that the shear rate associated with its settling is much less than the shear rate of the background flow $\left(w_{s} / a \ll \partial u / \partial z\right)$, the numerical results of Childs [21] confirm the dimensional reasoning above. However as the background flow weakens, there is a need to amend the settling rule and to include a settling term which is independent of $\partial u / \partial z$.

Here, we introduce a semi-empirical model for the settling speed, which was demonstrated by [21] to capture its full three-dimensional numerical evaluation and which encompasses the shear-rate dependence described above. We write

$$
w_{s}=\frac{\Delta \rho g a^{2}}{K_{n}}\left|\frac{\partial u}{\partial z}\right|^{1-n} w_{s a}+a\left(\frac{\Delta \rho g a}{K_{n}}\right)^{1 / n} w_{s b},
$$

where $w_{s a}$ and $w_{s b}$ are dimensionless constants, dependent on $n$. The two terms in (6) respectively represent the settling velocity in the regime of very large and vanishing shear rates in the externally imposed flow. Here we have simply summed the contributions from each regime to produce this empirical expression for the settling velocity. The regime of interest in this study corresponds to when the stress exerted by the particle due to its excess density is much less than the stress driving the channel flow, $\Delta \rho g a \ll G h$ and in this regime the settling velocity associated with the first term on the right hand side of (6) far exceeds the second term, except in regions where the velocity gradient vanishes. In fact in the analysis that follows, any dimensionally consistent relationship between the settling speed, the local shear and the properties of the particles and the fluid could be employed within the same framework without significantly altering the results; the key dependency is merely that the settling velocity decreases with decreasing shear rate.

We now identify the dimensionless variables. The characteristic streamwise velocity is based upon the pressure gradient, $G$, the consistency, $K_{n}$, and the flow 
depth $h$ and is given by $\mathcal{U}=\left(G h^{n+1} / K_{n}\right)^{1 / n}$, while the settling velocity scale is $\mathcal{W}=w_{s a} \Delta \rho g a^{2}(G h)^{(1-n) / n} / K_{n}^{1 / n}$. Vertical lengths are scaled with respect to the flow depth $h$ and horizontal lengths with respect to $h \mathcal{U} / \mathcal{W}$. Finally times are scaled by $h / \mathcal{W}$, the concentration field by $C_{0}$ and the deposit thickness by $h C_{0} /\left(1-\phi_{b}\right)$. Dimensionless variables are therefore defined by

$$
\left\{\hat{x}, \hat{z}, \hat{t}, \hat{u}, \hat{w}_{s}, \hat{\eta}, \hat{C}\right\}=\left\{\frac{x \mathcal{W}}{h \mathcal{U}}, \frac{z}{h}, \frac{\mathcal{W} t}{h}, \frac{u}{\mathcal{U}}, \frac{w_{s}}{\mathcal{W}}, \frac{h C_{0}}{\left(1-\phi_{b}\right)}, \frac{C}{C_{0}}\right\} .
$$

The governing equations are then given by

$$
\begin{aligned}
\frac{\partial}{\partial \hat{z}}\left(\left|\frac{\partial \hat{u}}{\partial \hat{z}}\right|^{n-1} \frac{\partial \hat{u}}{\partial \hat{z}}\right) & =-1, \\
\frac{\partial \hat{C}}{\partial \hat{t}}+\hat{u} \frac{\partial \hat{C}}{\partial \hat{x}}-\frac{\partial}{\partial \hat{z}}\left(\hat{w}_{s} \hat{C}\right) & =0, \\
\frac{\partial \hat{\eta}}{\partial \hat{t}} & =\hat{w}_{s}(0) \hat{C}(\hat{x}, 0, \hat{t}),
\end{aligned}
$$

where $\hat{w}_{s}=(\partial \hat{u} / \partial \hat{z})^{1-n}+\epsilon$ and $\epsilon=\left(\Delta \rho g a w_{s b}\right) /\left(G h w_{s a}\right)$. The latter is assumed to be small $(\epsilon \ll 1)$ and potentially dependent on $n$. The concentration field initially vanishes $(\hat{C}(\hat{x}, \hat{z}, 0)=0)$ and for $\hat{t}>0$, a vertically uniform source is imposed, $\hat{C}(0, \hat{z}, \hat{t})=1$. The velocity field is subject to boundary conditions at the top $(\hat{z}=1)$ and bottom $(\hat{z}=0)$ of the flow, which are problem-specific and will be introduced when needed. The deposit initially vanishes $(\hat{\eta}(\hat{x}, 0)=0)$ and under the assumption that the deposit depth is much less that the channel depth, we have evaluated the settling flux at $z=0$, rather than $z=\eta$. It is also useful to define the steady concentration field, $\hat{C}_{\infty}(\hat{x}, \hat{z})$, which is attained after the initial transient has decayed. From henceforth we drop the caret notation and will assume, unless stated otherwise, that all variables are dimensionless according to the definitions in (7).

\subsection{Construction of solution}

We form the solution for the concentration field, $C(x, z, t)$, and the deposit, $\eta(x, t)$, by taking the Laplace transform with respect to $t$. Thus we define

$$
\bar{C}(x, z, p)=\int_{0}^{\infty} C(x, z, t) \mathrm{e}^{-p t} \mathrm{~d} t,
$$

and the governing equation (9) becomes

$$
p \bar{C}+u(z) \frac{\partial \bar{C}}{\partial x}-w_{s} \frac{\partial \bar{C}}{\partial z}-\frac{\partial w_{s}}{\partial z} \bar{C}=0,
$$

subject to $\bar{C}(0, z, p)=1 / p$. We now introduce characteristics parameterised by $s$ and $\xi$ such that (12) may be written as

$$
\begin{aligned}
& \frac{\mathrm{d} x}{\mathrm{~d} s}=u(z), \quad \frac{\mathrm{d} z}{\mathrm{~d} s}=-w_{s}(z), \quad \frac{\mathrm{d} \bar{C}}{\mathrm{~d} s}=-p \bar{C}+\frac{\mathrm{d} w_{s}}{\mathrm{~d} z} \bar{C}, \\
& \text { subject to } x=0, z=\xi \text { and } \bar{C}=1 / p \text { at } s=0 \text { for } 0<\xi<1 .
\end{aligned}
$$


It is straightforward to integrate (13) subject to (14) to yield

$$
\bar{C}=\frac{1}{p} \exp \left(-p s+\int_{0}^{s} \frac{\mathrm{d} w_{s}}{\mathrm{~d} z} \mathrm{~d} s\right) .
$$

Inverting the Laplace transform then gives

$$
C(x, z, t)=\mathcal{H}(t-s) \frac{w_{s}(\xi)}{w_{s}(z)}
$$

where the characteristic variables $s$ and $\xi$ are functions of $x$ and $z$ and are implicitly given by (13) and its associated boundary condition (14), and $\mathcal{H}(s)$ is the Heaviside step function. The dimensionless deposit is given by

$$
\eta(x, t)=\int_{0}^{t} \mathcal{H}(t-s) w_{s}(\xi) \mathrm{d} t
$$

and we will sometimes plot $\eta(x, t) / t$ as a function of $x$, noting that

$$
\frac{\eta(x, t)}{t} \rightarrow w_{s}(0) C_{\infty}(x, 0) \quad \text { as } \quad t \rightarrow \infty .
$$

We observe from (16) that settling through Newtonian fluid, for which the settling velocity is constant, yields a concentration of particles that either vanishes (if $s(x, z)>t$ ) or takes the value unity (if $s(x, z)<t$ ); the latter occurs at locations reached by the advected suspension. Conversely for a non-Newtonian fluid, the concentration field varies spatially, thus illustrating immediately the control that the rheology of the interstitial fluid has on the suspension and the distribution of the deposit.

\section{Free-surface flows}

We now apply our formulation to free-surface flows driven by a constant pressure gradient. The dimensionless velocity field satisfies a no-slip condition $(u(0)=0)$ and vanishing shear stress at the free surface $(\partial u / \partial z(1)=0)$; it is given by

$$
u=\frac{n}{1+n}\left(1-(1-z)^{(1+n) / n}\right)
$$

while the settling velocity is then given by

$$
w_{s}=(1-z)^{(1-n) / n}+\epsilon
$$

The characteristics in terms of the parameters $s$ and $\xi$ are determined by

$$
\int_{z}^{\xi} \frac{\mathrm{d} z^{\prime}}{w_{s}\left(z^{\prime}\right)}=s \quad \text { and } \quad x=\int_{z}^{\xi} \frac{u\left(z^{\prime}\right)}{w_{s}\left(z^{\prime}\right)} \mathrm{d} z^{\prime} .
$$

The concentration field is provided by (16). It is insightful to plot the characteristic curves in the $(x, z)$ plane and examples for $n=1 / 3$ and $2 / 3$ are 

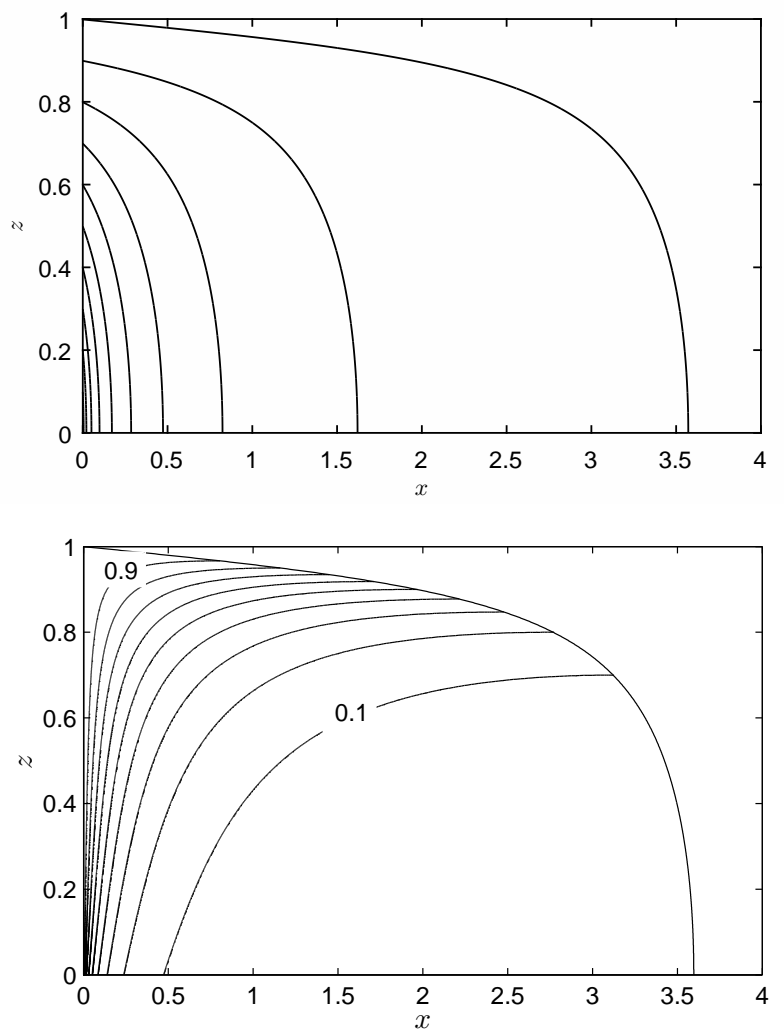

Figure 1: (a) The characteristics in the $(x, z)$ plane; and (b) contours of the steady-state concentration field for a sustained release of particles from $x=0$ in fluid with power-law index $n=1 / 3$ and settling parameter $\epsilon=0.01$. Characteristics are plotted for $\xi=0.1-1$ (with intervals of 0.1 ) and the contours of $C_{\infty}$ are plotted for $C_{\infty}=0.1-0.9$ (with intervals of 0.1 ). The characteristic from $z=1$ (corresponding to $\xi=1$ ) bounds the region within which the concentration field is non-vanishing. 

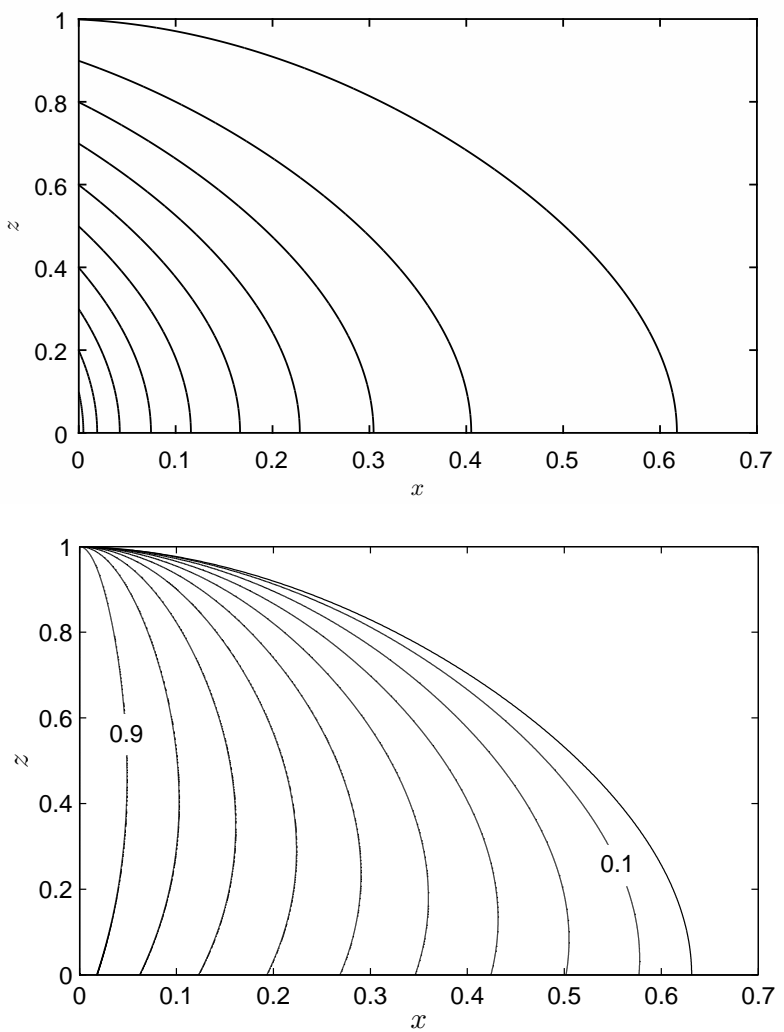

Figure 2: (a) The characteristics in the $(x, z)$ plane; and (b) contours of the steady-state concentration field for a sustained release of particles from $x=0$ in fluid with power-law index $n=2 / 3$ and settling parameter $\epsilon=0.01$. Characteristics are plotted for $\xi=0.1-1$ (with intervals of 0.1 ) and the contours of $C_{\infty}$ are plotted for $C_{\infty}=0.1-0.9$ (with intervals of 0.1 ). The characteristic from $z=1$ (corresponding to $\xi=1$ ) bounds the region within which the concentration field is non-vanishing. 
plotted in figures 1 and 2. In these figures we immediately note the effect of the non-Newtonian rheology of the suspending fluid. As commented above, for a Newtonian fluid $(n=1)$, the concentration field either vanishes or adopts the scaled source value of unity. In contrast for shear-thinning fluids $(n<1)$, the concentration varies spatially. This arises because the settling velocity is shear-rate dependent and in these shear flows for which the the shear rate is spatially variable, the settling velocity also varies spatially. In particular, close to the free surface, where the shear rate vanishes, the settling velocity is very strongly diminished and this has a very significant effect on the concentration field when $n \leq 1 / 2$. In fact we note from figures 1 and 2 that the concentration field may increase with distance from the underlying boundary in some or all of the flow even though it was vertically uniform at source, which has the potential to develop gravitationally-induced overturning as the flow develops. We may calculate the vertical gradient of the steady concentration field from (16)

$$
\frac{\partial C_{\infty}}{\partial z}=\frac{w_{s}^{\prime}(\xi)}{w_{s}(z)} \frac{\partial z}{\partial \xi}-\frac{w_{s}(\xi)}{w_{s}(z)^{2}} w_{s}^{\prime}(z)
$$

Then finding $\partial \xi / \partial z$ from (21) we find that

$$
\frac{\partial C_{\infty}}{\partial z}=\frac{-n^{2} w_{s}(\xi) u(z)}{\left(1-n^{2}\right) w_{s}(z)^{2}}\left(\frac{1}{F(\xi)}-\frac{1}{F(z)}\right)
$$

where $F(z)=-\left(1-n^{2}\right) u(z) / w_{s}^{\prime}(z) n^{2}=(1-z)^{(2 n-1) / n}-(1-z)^{3}$. Since $0 \leq z \leq \xi \leq 1$, static instability, $\partial C_{\infty} / \partial z>0$, then requires either $n \leq 1 / 2$ or when $n>1 / 2$ that $z$ is sufficiently small. Thus we deduce that for strongly shear thinning fluids $(n \leq 1 / 2)$, the concentration field exhibits a positive vertical gradient throughout the entire domain (figure 1). However for more weakly shear-thinning fluids $(n>1 / 2)$, there is only a positive gradient of concentration in the lower region of the flow (figure 2).

We may evaluate the maximum downstream distance reached by the settling particles; this dimensionless distance is given by

$$
\begin{aligned}
x_{f m} & =\int_{0}^{1} \frac{u\left(z^{\prime}\right)}{w_{s}\left(z^{\prime}\right)} \mathrm{d} z^{\prime} \\
& =\frac{n}{n+1}\left(\frac{1}{\epsilon} F_{21}\left(1, \alpha ; 1+\alpha ;-\frac{1}{\epsilon}\right)+\frac{1}{3} F_{21}\left(1,3 \alpha ; 1+3 \alpha ;-\frac{1}{\epsilon}\right)-\frac{1}{3}(24) 5\right)
\end{aligned}
$$

where $\alpha=n /(1-n)$ and $F_{21}$ is a hypergeometric function [22]. This location corresponds to where the characteristic emanating from $(x, z)=(0,1)$ reaches the lower boundary and it is a function of the flow-index $n$ and the settling parameter $\epsilon$. It is insightful to draw out the leading-order asymptotic dependence 


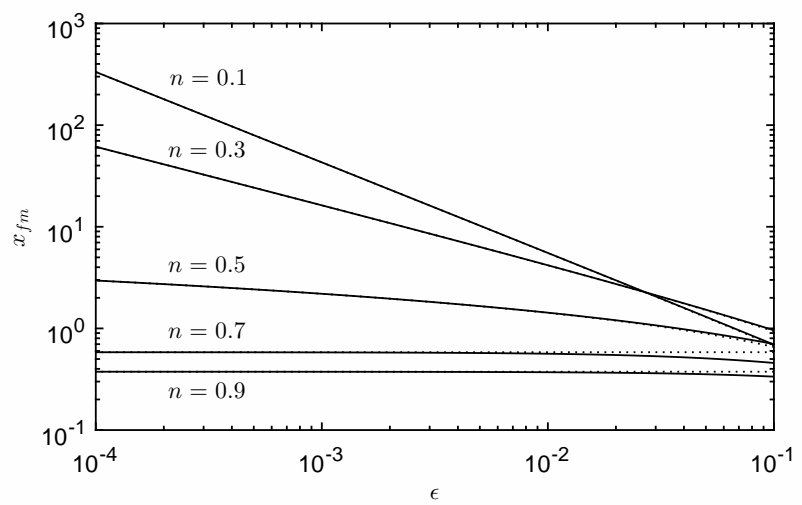

Figure 3: The maximum distance propagated by the suspension in a free-surface flow, $x_{f m}$, as a function of the quiescent settling parameter, $\epsilon$, for fluids with power-law indices $n=$ $0.1,0.3,0.5,0.7$ and 0.9. Also plotted are the leading order asymptotic predictions of this distance (dotted lines), although these are often overlain by the exact results.

of $x_{f m}$ upon the parameter $\epsilon$, which correct to $O(1)$ is given by

$$
x_{f m}= \begin{cases}\frac{n}{n+1}\left(\frac{n \pi \epsilon^{(2 n-1) /(1-n)}}{(1-n) \sin (n \pi /(1-n))}+\frac{n}{2 n-1}-\frac{1}{3}\right), & n<1 / 2, \\ \frac{1}{3}\left(\log \left(\frac{1}{\epsilon}\right)-\frac{1}{3}\right), & n=1 / 2, \\ \frac{n}{n+1}\left(\frac{n}{2 n-1}-\frac{1}{3}\right), & n>1 / 2 .\end{cases}
$$

The numerically evaluated $x_{f m}$ and its leading order asymptotic representation are plotted in figure 3, noting that unless $\epsilon$ is relatively large then the two are indistinguishable in this figure. This calculation brings out clearly that the maximum distance propagated from source is flow index dependent and crucially for strongly shear-thinning suspending fluids $(n \leq 1 / 2)$, the maximum distance depends strongly on the quiescent settling parameter, $\epsilon$. Indeed when $n \leq 1 / 2$, $x_{f m} \rightarrow \infty$ as $\epsilon \rightarrow 0$, a result that emerges because the time for a particle to settle away from the free-surface, where the shear rate vanishes, becomes unbounded in this limit. We may also compute $t_{f m}$, which is defined to be the time at which the suspension first reaches $x_{f m}$. This time is given directly from the characteristic equations; it corresponds to $t_{f m}=s\left(x_{f m}, 0\right)$ and may be expressed as

$$
t_{f m}=\int_{0}^{1} \frac{1}{\eta^{(1-n) / n}+\epsilon} \mathrm{d} \eta=\frac{1}{\epsilon} F_{21}\left(1, \alpha ; 1+\alpha ;-\frac{1}{\epsilon}\right) .
$$

Finally we plot the growth of the deposit scaled by the time, $\eta(x, t) / t$ as a function of distance at various instances of $t / t_{f m}$ (figure 4 ). As $t \rightarrow \infty$, 

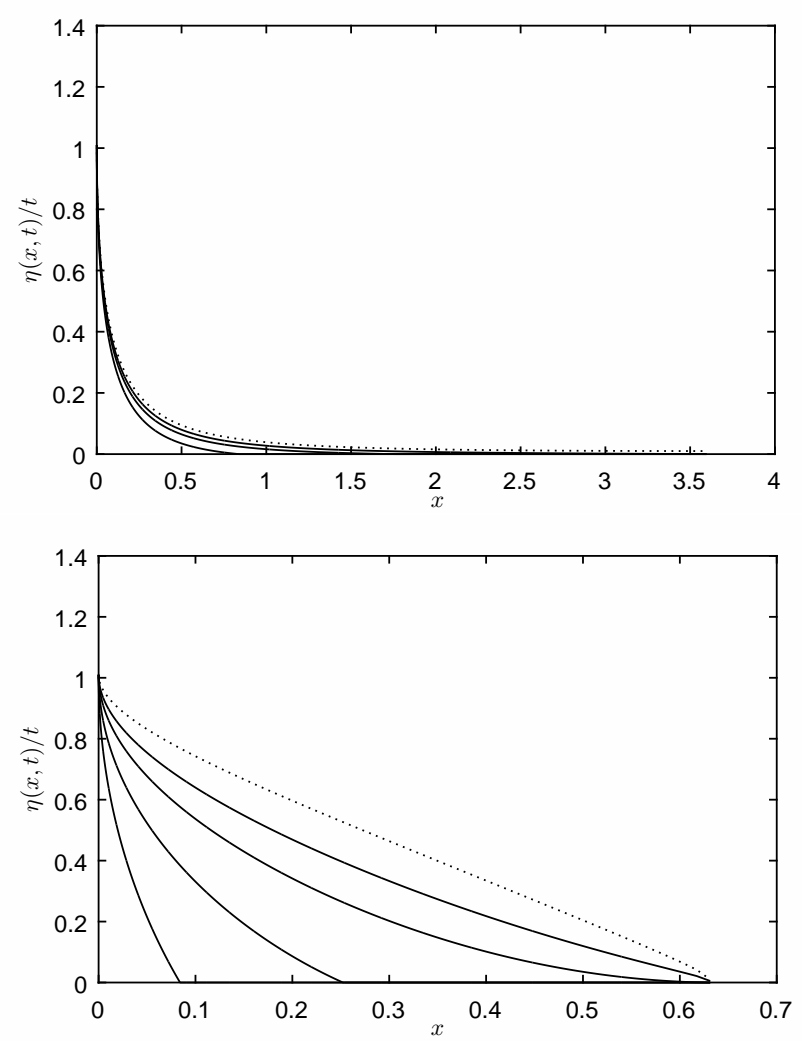

Figure 4: The temporal development of the thickness of the deposit as a function of distance for (a) $n=1 / 3$ and (b) $n=2 / 3$. The ratio of the accumulated deposit to time $\eta(x, t) / t$ is plotted in $(\mathrm{a}, \mathrm{b})$ at $t / t_{f m}=0.25,0.5,1$ and additional at $t / t_{f m}=2$ in (b), where $t_{f m}$ is the time at which the flow first reaches its maximum extent $\left(x_{f m}\right)$. Also plotted is the long-time asymptote, $w_{s}(0) C_{\infty}(x, 0)$ (dotted lines).

then $\eta(x, t) / t \rightarrow w_{s}(0) C_{\infty}(x, 0)$ but the steady state may be approached only gradually. From figure 4 we again note the strong effect of the non-Newtonian rheology of the suspending fluid. For the Newtonian case $(n=1)$, the steadystate deposit is spatially uniform for $x<x_{f m}$, but for shear-thinning fluids, the steady-state deposit progressively thins with downstream distance due to the inhibited settling velocity of the particles close to the free surface (figure 4). A useful additional measure of the deposits' extent is the distance from source by which half of the source flux of particles have settled out of the flow to the underlying boundary, $x_{f b}$. This distance is determined by finding the elevation at source of the characteristic below which the fluid motion carries half of the 
fluid flux; $z_{*}$ is given by

$$
\int_{0}^{z_{*}} u\left(z^{\prime}\right) \mathrm{d} z^{\prime}=\frac{1}{2} \frac{n}{2 n+1}
$$

The median position within the deposit, $x_{f b}$ is then given by

$$
x_{f b}=\int_{0}^{z_{*}} \frac{u\left(z^{\prime}\right)}{w_{s}\left(z^{\prime}\right)} \mathrm{d} z^{\prime} .
$$

It is immediately evident that for Newtonian fluids the median distance in the deposit is half of the maximum extent of the deposit, $x_{f b}=x_{f m} / 2$, but for non-Newtonian fluids this distance is straightforwardly determined numerically from (29) to find that $x_{f b} / x_{f m}<1 / 2$.

\section{Channel flow}

We now analyse the concentration distribution for two-dimensional flow along a rigid, horizontal channel, driven by a constant pressure gradient. No-slip conditions are now enforced at the boundaries $z=0,1$ and the velocity profile is given by

$$
u=\frac{n}{1+n}\left(\left(\frac{1}{2}\right)^{(1+n) / n}-\left|\frac{1}{2}-z\right|^{(1+n) / n}\right) .
$$

The dimensionless settling velocity is given by

$$
w_{s}=\left|\frac{1}{2}-z\right|^{(1-n) / n}+\epsilon .
$$

The key feature of the behaviour of suspensions in these channel flows is due to the velocity maximum at $z=1 / 2$. At this elevation the local shear rate vanishes and thus the settling velocity is minimised. This elevation therefore acts as a 'kinematic barrier' to the settling particles; particles that are released in the upper half of the flow must settle across this barrier in order to form the deposit. Since the settling velocity is minimised at the velocity maximum, this elevation and the settling velocity in its region exert control on the structure of the underlying deposit. There are two important consequences: the concentration field close to the velocity maximum may increase above its initial value at source due to the slow passage of particles across the kinematic barrier and the deposit on the underlying boundary may not decrease monotonically with distance from source. We emphasise that these flows are purely depositional and that these features arise due to the non-Newtonian controls on the settling velocity. It is also noteworthy that this phenomenon will occur in any flow with a velocity maximum; for example, fully developed flows, driven by a constant pressure gradient, could exhibit a velocity maximum if they satisfy no slip at their base and experience a resistive stress at their top surface.

We compute the characteristics that emerge from the source and the steady concentration field $C_{\infty}(x, z)$ for $n=1 / 3$ and $n=2 / 3$ in figure 5 and 6 . We 
observe a clustering of the characteristics around the velocity maximum due to the reduced settling velocity and associated with this phenomenon is an elevated concentration field. Eventually the particles do settle across the velocity maximum, but the effects of the kinematic barrier manifest themselves in the concentration field at the base of the flow and thus influence the distribution of the deposit (see figures 5 and 6 ).

It is straightforward to deduce the maximum and minimum values of the steady-state concentration field, $C_{\infty}$. First, the maximum occurs on the characteristic labelled by $\xi=1$ at the velocity maximum $(z=1 / 2)$. It is therefore given by

$$
C_{\max }=\frac{w_{s}(1)}{w_{s}(1 / 2)}=\frac{2^{(n-1) / n}+\epsilon}{\epsilon} .
$$

The minimum occurs on the characteristic labelled by $\xi=1 / 2$ at the base of the flow $(z=0)$ and is equal to

$$
C_{\min }=\frac{w_{s}(1 / 2)}{w_{s}(0)}=\frac{\epsilon}{2^{(n-1) / n}+\epsilon}=\frac{1}{C_{\max }} .
$$

For $\epsilon=0.01$ and $n=2 / 3,\left(C_{\max }, C_{\min }\right)=(71.7107,0.0139)$, while for $n=1 / 3$, $\left(C_{\max }, C_{\min }\right)=(26,0.0385)$. It is thus apparent the presence of this kinematic barrier can lead to very significant amplification of the source concentration as settling is slowed close to the velocity maximum.

The maximum distance propagated by the suspension is still given by (24), but due to the symmetry of the fluid field about $z=1 / 2$, this may be written

$$
x_{c m}=\frac{2 n}{n+1}\left(\int_{0}^{1 / 2} \frac{\left(\frac{1}{2}\right)^{(n+1) / n}+\epsilon \eta^{2}}{\eta^{(1-n) / n}+\epsilon} \mathrm{d} \eta-\frac{1}{24}\right) .
$$

When $\epsilon \ll 1$, an asymptotic representation of $x_{m}$ up to $O(\epsilon)$ is given by

$$
x_{c m}= \begin{cases}\frac{2 n}{n+1}\left(\frac{2^{-(1+n) / n} n \pi \epsilon^{(2 n-1) /(1-n)}}{(1-n) \sin (n \pi /(1-n))}+\frac{n}{8(2 n-1)}-\frac{1}{24}\right), & n<1 / 2, \\ \frac{1}{3}\left(\frac{1}{4} \log \left(\frac{1}{2 \epsilon}\right)-\frac{1}{12}\right), & n=1 / 2, \\ \frac{2 n}{n+1}\left(\frac{n}{8(2 n-1)}-\frac{1}{24}\right), & n>1 / 2 .\end{cases}
$$

Both the exact evaluation of (34) and the asymptotic representation (35) are plotted in figure 7 , noting that the asymptotic formula provides an accurate expression for the exact form when $\epsilon \ll 1$. As in $\S 3$, this dependence draws out the very significant role that the kinematic barrier plays in determining $x_{c m}$ when the fluid is strongly shear thinning (i.e. for $n<1 / 2, x_{c m} \rightarrow \infty$ as $\epsilon \rightarrow 0)$. For less strongly shear-thinning fluids $(n>1 / 2)$ there is nevertheless a dependence on $\epsilon$, but the maximum extent remains bounded as $\epsilon \rightarrow 0$. The earliest time at which the suspended particles reach the maximum extent is 

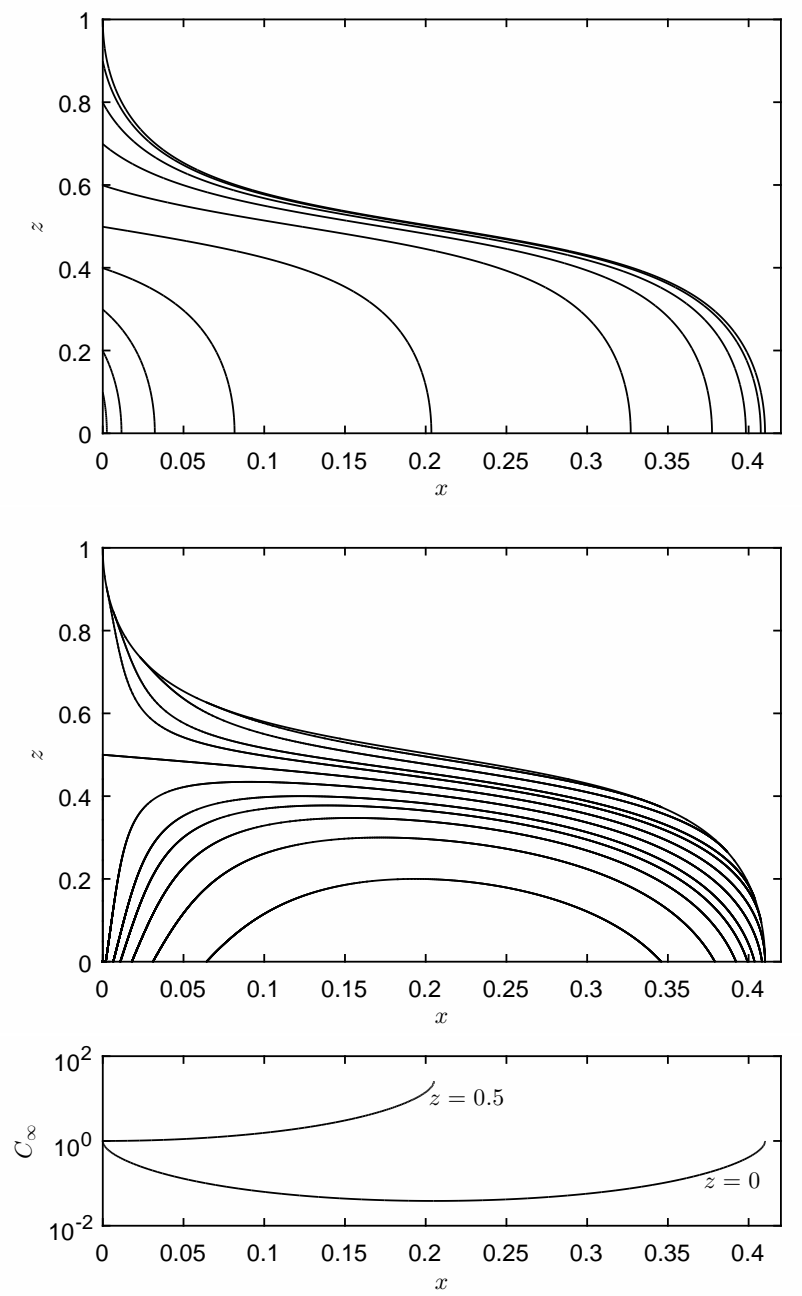

Figure 5: (a) The characteristics in the $(x, z)$ plane; (b) contours of the steady-state concentration field; and (c) the steady-state concentration field, $C_{\infty}$, as a function of downstream distance, $x$, at $z=0$ and $z=0.5$ for a sustained release of particles from $x=0$ in fluid with power-law index $n=1 / 3$ and settling parameter $\epsilon=0.01$ flowing within a horizontal channel. Characteristics are plotted for $\xi=0.1-1$ (with intervals of 0.1 ) and the contours of $C_{\infty}$ are plotted for $C_{\infty}=0.1,0.2,0.3,0.4,0.5,0.7,1,1.5,2,4,10$. The characteristic from $z=1$ (corresponding to $\xi=1$ ) bounds the region within which the concentration field is non-vanishing. 

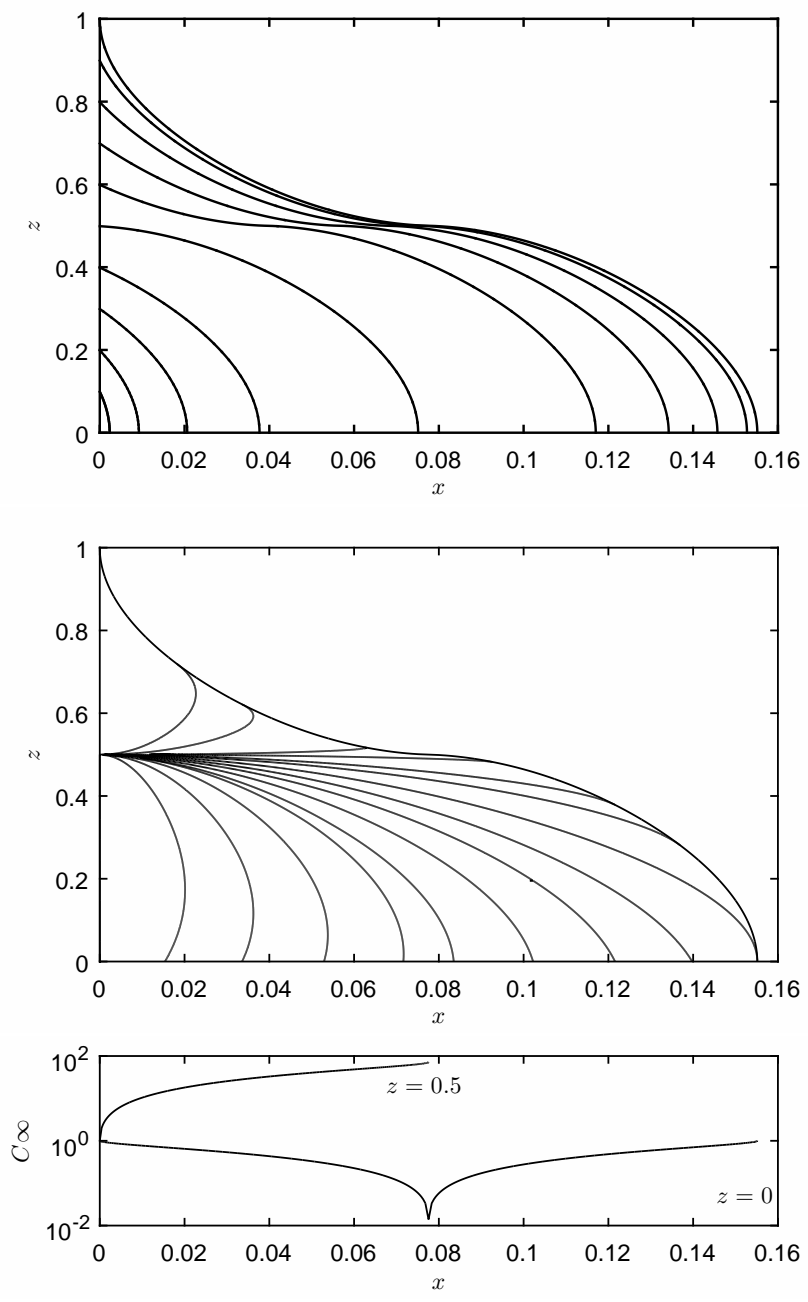

Figure 6: (a) The characteristics in the $(x, z)$ plane; (b) contours of the steady-state concentration field and (c) the steady-state concentration field, $C_{\infty}$, as a function of downstream distance, $x$, at $z=0$ and $z=0.5$ for a sustained release of particles from $x=0$ in fluid with power-law index $n=2 / 3$ and settling parameter $\epsilon=0.01$ flowing within a horizontal channel. Characteristics are plotted for $\xi=0.1-1$ (with intervals of 0.1 ) and the contours of $C_{\infty}$ are plotted for $C_{\infty}=0.1,0.3,0.5,0.7,1,1.5,2,5$. The characteristic from $z=1$ (corresponding to $\xi=1$ ) bounds the region within which the concentration field is non-vanishing. 


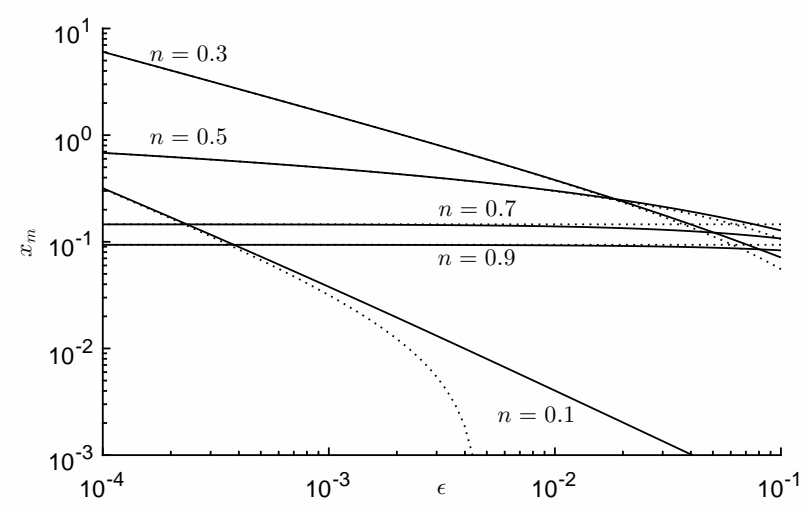

Figure 7: The maximum distance propagated by the suspension in a channel flow, $x_{c m}$, as a function of the quiescent settling parameter $\epsilon$ for fluids with power-law indices $n=$ $0.1,0.3,0.5,0.7$ and 0.9. Also plotted are the leading order asymptotic predictions of this distance (dotted lines).

given by

$$
t_{c m}=\int_{0}^{1} \frac{1}{\left|\frac{1}{2}-z\right|^{(1-n) / n}+\epsilon} \mathrm{d} z=2^{(1-n) / n} \int_{0}^{1} \frac{1}{\eta^{(1-n) / n}+2^{(1-n) / n} \epsilon} \mathrm{d} \eta .
$$

We may now evaluate the ratio of the maximum distance propagated by the particles settling within a channel flow to the maximum distance propagated within a free-surface flow, $X_{R}=x_{c m} / x_{f m}$, and the ratio of the times that it takes to propagate to this distance, $T_{R}=t_{c m} / t_{f m}$. We comment that the distance propagated in channel flows is always less than that in free-surface flows $\left(X_{R}<1\right)$. This reflects the role of the no-slip boundary condition at the upper surface of the channel, which reduces the horizontal flow speed relative to the free-surface flow for the same driving pressure gradient. However, the time taken for the suspension to travel this distance is always higher for the channel flows because the sedimenting particles must pass fully across the kinematic barrier at the velocity maximum $\left(T_{R}>1\right)$. In the regime $\epsilon \ll 1$, we find that

$$
X_{R}=\left\{\begin{array}{cc}
2^{-1 / n}, & n \leq 1 / 2, \\
1 / 4, & 1 / 2<n,
\end{array} \quad \text { and } \quad T_{R}=\left\{\begin{array}{cc}
2, & n \leq 1 / 2, \\
2^{(1-n) / n}, & 1 / 2<n .
\end{array}\right.\right.
$$

Finally we analyse the deposit formed by these channel flows (see figure 8). The most significant feature here is that the depth of the deposit scaled by time since release, $\eta(x, t) / t$, is no longer monotonically decreasing with distance from source. Instead there is a local minimum due to suppression of settling as the particles encounter the kinematic barrier at the velocity maximum, after which the deposit thickens towards its maximum streamwise extent.

As for the free-surface flows, we may determine the median distance within the deposit, $x_{c b}$, which is the distance from source by which half of the source 

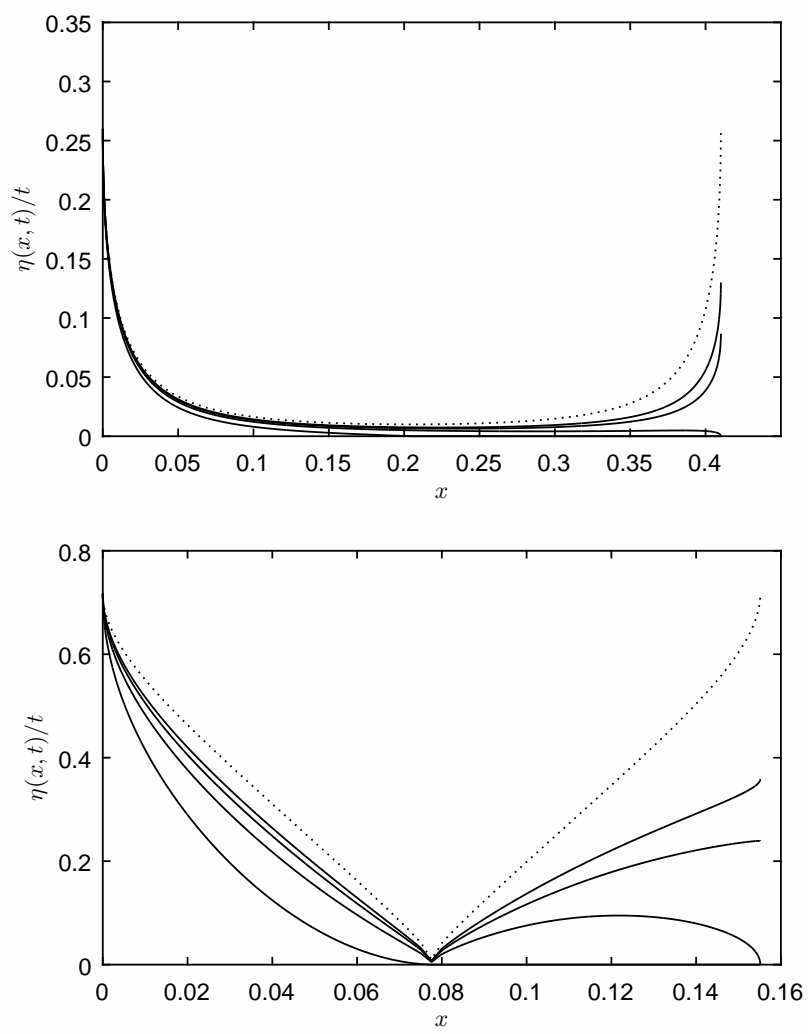

Figure 8: The temporal development of the deposit as a function of distance for (a) $n=1 / 3$ and (b) $n=2 / 3$. The ratio of the accumulated deposit to time $\eta(x, t) / t$ is plotted at $t / t_{c m}=$ $0.25,0.5,1,2$, where $t_{c m}$ is the time at which the flow first reaches its maximum extent $\left(x_{c m}\right)$. Also plotted is the long-time asymptote, $w_{s}(0) C_{\infty}(x, 0)$ (dotted lines). 


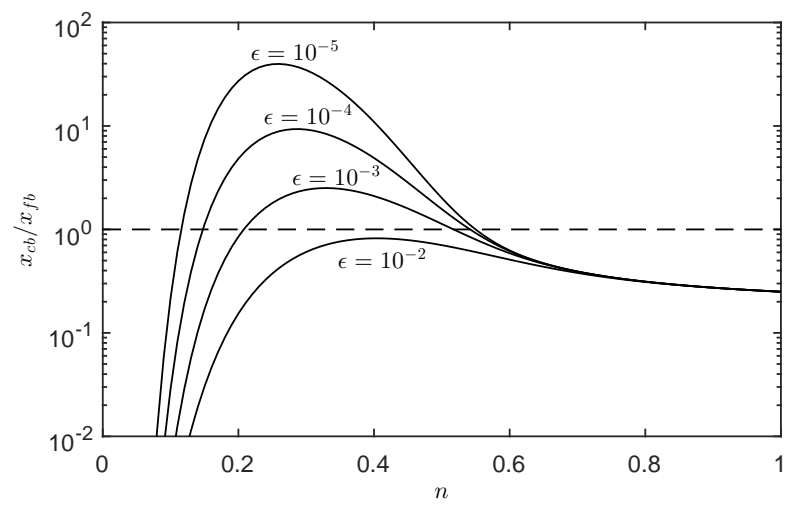

Figure 9: The ratio of the median distance of the deposit from source in a channel flow to that in a free-surface flow, $x_{c b} / x_{f b}$ as a function of flow index for different values of the quiescent settling parameter.

flux of particles has settled to the underlying boundary. For channel flows, this corresponds to the position at the boundary associated with the characteristic emanating from $z=1 / 2$, thus giving $x_{c b}=x_{c m} / 2$. It is then interesting to examine the ratio of the median distance within the deposit in a channel flow to the median distance in a free-surface flow, $x_{c b} / x_{f b}$, which is plotted in figure 9 . Here we note that for weakly shear-thinning fluids, this ratio is less than unity, but that it exceeds unity for more strongly shear-thinning fluids. Thus although channel flows do not lead to the suspension propagating further than their freesurface counterparts, they may nevertheless lead to the median position in the deposit being further from source.

\section{Bidisperse suspension}

In this section we analyse the evolution of a bidisperse suspension released continuously from the source at $x=0$ in a two-dimensional channel. The suspension comprises two classes of particles, which differ only in size; the ratio of the diameter of species 2 to the diameter of species 1 is denoted by $\sqrt{\lambda}$ and so that when non-dimensionalised using the properties of species 1 , the settling velocities are given by

$$
w_{s 1}=\left(\frac{\partial u}{\partial z}\right)^{1-n}+\epsilon \quad \text { and } \quad w_{s 2}=\lambda\left(\frac{\partial u}{\partial z}\right)^{1-n}+\lambda^{(1+n) / 2 n} \epsilon
$$

The suspension is assumed to be dilute so that each species evolves independently, and in this illustrative calculation, we further assume that the initial concentrations at the source are equal and uniform throughout the depth of the flow. 
We compute the settling flux of each species into the deposit, since this determines its composition. The steady-state settling flux is given by

$$
F_{b i}(x)=w_{s i}(0) C_{\infty i}(x, 0)=w_{s i}\left(\xi_{i}(x, 0)\right),
$$

where $i=1,2$, corresponding to each of the classes of particles. The characteristic label $\xi_{i}(x, 0)$ is defined by

$$
\int_{0}^{\xi_{i}(x, 0)} \frac{u}{w_{s i}} \mathrm{~d} z=x .
$$

It corresponds to the characteristic from starting height $\xi_{i}$ which reaches $z=0$ at distance $x$ from the source. We compute the proportion, $\psi(x)$ of the settling flux that corresponds to the larger ('coarse') particles since $\lambda<1$; it is given by

$$
\psi(x)=\frac{F_{b 1}(x)}{F_{b 1}(x)+F_{b 2}(x)}
$$

with $\psi=1$ corresponding to $100 \%$ coarse particles in the deposit and $\psi=0$ to $100 \%$ fine particles.

We evaluate the proportion of coarse particles for bidisperse suspension in flows with power-law index $n=1 / 3,2 / 3$ and 1 for $\lambda=1 / 2$ (see figure 10 ). We note that for all three flows $\psi(0)=2 / 3+\ldots$ when $\epsilon \ll 1$, since this corresponds to the settling flux from the base of the flow at the source. However further downstream the distribution of the deposit depends on the power-law flow index. For Newtonian fluids $(n=1)$, the deposit exhibits two compositions. Initially, close to the source, there is a mixture of coarse and fine particles $(\psi=2 / 3)$ and this proportion does not vary until all of the larger particles have settled out. Thereafter the deposit comprises only fine particles. This distribution featuring two uniform regions is found because for a Newtonian fluid the settling velocity of each class is spatially uniform.

The distribution of the composition within the deposit for non-Newtonian suspension, however, exhibits more complicated variations. The settling flux of the coarse fraction initially decreases with distance from the source due to the inhibition of the settling velocity as the coarse particle sediment from regions with reduced local shear rates. This leads to a reduction of the proportion, $\psi(x)$. However the proportion of coarse particles is minimised at the location where the characteristic from the velocity maximum first reaches the bed. Thereafter the proportion, $\psi(x)$ increases with distance downstream until the location is reached which corresponds to the maximum distance for the coarse class. Further downstream the deposit only consists of fine particles.

This complicated pattern of fining and then coarsening with downstream distance is due entirely to the control that the non-Newtonian rheology imparts on the settling velocity of each class; there is no need to invoke more exotic physical mechanisms, such as kinetic sieving and bed remobilisation [5]. 

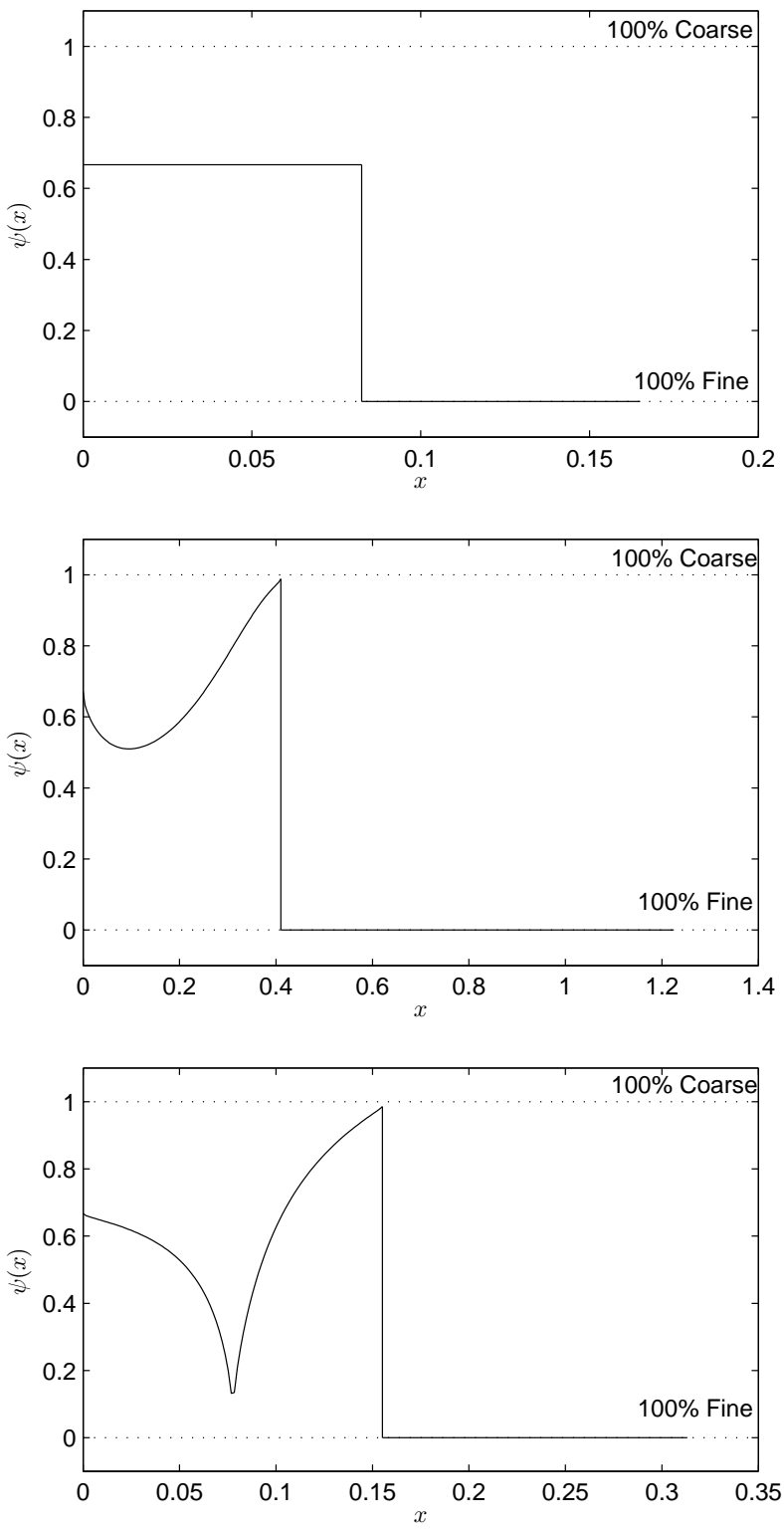

Figure 10: The steady-state proportion of coarse particles in the deposit from a sustained release of a bidisperse mixture with particle size in the ratio $2^{-1 / 2}$ and initial equal volume fractions of each species in a horizontal channel flow for (a) $n=1$; (b) $n=1 / 3$; and $n=2 / 3$. 


\section{Yield stress}

To examine the effects of a yield stress on the dynamic settling processes and the kinematic barrier to settling in regions of low shear rates, we analyse the evolution of a suspension of relatively dense particles within the flow of a Herschel-Bulkley fluid in a horizontal channel. We scale the dimensional variables in the same way as $\S 2$, where $K_{n}$ is now the consistency parameter in the Herschel-Bulkley model. In this problem, however, there is an important additional dimensionless parameter, $B=\sigma_{Y} /(G h)$, which measures the magnitude of the yield stress, $\sigma_{Y}$, relative to the stress exerted by the imposed pressure gradient. Flow occurs if $B<1 / 2$ and the fully developed velocity field is given by

$$
u=\frac{n}{1+n} \begin{cases}\left(\frac{1}{2}-B\right)^{(n+1) / n}-\left(\left|\frac{1}{2}-z\right|-B\right)^{(n+1) / n}, & |z-1 / 2|>B \\ \left(\frac{1}{2}-B\right)^{(n+1) / n}, & |z-1 / 2|<B .\end{cases}
$$

In terms of the dimensionless variables adopted here, there is an unyielded region of width $2 B$ centred around the middle of the channel. Within this region $(|z-1 / 2|<B)$ the shear rate vanishes and thus the existence and influence of the kinematic barrier will be different from what was elucidated in $\S 4$ where the shear rate vanished only at $z=1 / 2$.

Particles are assumed to be sufficiently massive that they settle through the 'unyielded' region at a small velocity [11]; they settle through the yielded region at a rate dependent upon the background shear rate, which sets the 'local' viscosity. A simple expression for the dimensionless settling velocity, which captures this effect, is given by

$$
w_{s}=\left(\frac{\partial u}{\partial z}\right)^{1-n}+\epsilon= \begin{cases}\left(\frac{1}{2}-B-z\right)^{1 / n-1}+\epsilon, & |z-1 / 2|>B \\ \epsilon, & |z-1 / 2|<B\end{cases}
$$

although the interpretation of $\epsilon$ is now different from the case of vanishing yield stress $(c f . \S 2)$. This settling law is of course a very simple expression for the full dynamics. Equation (43) captures the dependence of the effective viscosity (and hence the settling speed) upon the local shear rate $(\partial u / \partial z)$ when the dimensionless shear is large and becomes independent of the local shear rate when it becomes small. Dimensional analysis asserts that there are now two dimensionless ratios characterising the particle motion if inertial effects are neglected, namely $\Delta \rho g a /(G h)$ and $\Delta \rho g a / \sigma_{Y}$ and the latter of these can not be vanishingly small or else the particle would not settle through the unyielded region. More accurate expressions for the settling velocity could of course be employed when they become available, but the important dynamical controls are encompassed within this simple expression — and here we explore their consequences.

We calculate the steady-state concentration, $C_{\infty}$, using the method of characteristics as in $\S 2.1$. We note that the concentration field is given by (16), in 
which the dimensionless settling velocity is given by (43). The characteristics are determined by (13) using the dimensionless flow velocity (42) and settling velocity (43).

For strongly shear-thinning fluids $(n<1 / 2)$, we find that the effects of a yield stress are relatively minor. The characteristics that pass through the unyielded region now feature a linear section and the associated concentration field then develops a region within which the concentration adopts its initial value (see figure 11, cf. figure 5). This region is attached to the source $(x=0)$ and within the plug $(|z-1 / 2|<B)$, where the settling velocity is constant. The introduction of the yield stress reduces the fluid velocity within the channel and this could lead to reduced runout distances. However the yield stress also introduces an unyielded portion of the flow through which the particles settle slowly. This broadening of the effective kinematic barrier increases the settling times and thus increases the runout distances. Which of these processes dominate depends upon the values of $B$ and $\epsilon$; for $n=1 / 3, B=0.2$ and $\epsilon=10^{-2}$ (as in figure 11), we note that the runout length is reduced by the introduction of the yield stress.

For weakly shear-thinning fluids $(1 / 2<n<1)$, however, the effects are much more significant. Without a yield stress $(B=0)$, although the particles are retarded as they settle through the kinematic barrier, their transit time across this zone remains bounded and independent of $\epsilon$ when $\epsilon \rightarrow 0$. This is not the case for flows with a yield stress, for which the time to settle across the unyielded plug is proportional to $B / \epsilon$. This has a profound effect upon the characteristics, the concentration field and the depositional flux to the underlying boundary (see figures 6 and 12). The characteristics now feature an extended region over which they vary linearly with distance downstream, reflecting the transit through the unyielded region. The progress through this zone is much slower than through the yielded regions that sandwich it. The concentration field shows the consequences of this effect. There is a relatively large region $\left(\mathcal{D}_{2}\right.$ in figure 12) within which the concentration is very close to its initial value. This corresponds to the slow settling of particles through the plug. Below it (region $\mathcal{D}_{1}$ ), the concentration rapidly diminishes with distance downstream due to rapid settling of particles close to the source, which are not replenished from above until much further downstream. The uppermost region $\left(\mathcal{D}_{3}\right)$ is narrow and exhibits high concentrations; particles settle quickly through the yielded region $(z-1 / 2>B)$, accumulate near the upper boundary of the unyielded region and then settle through it (slowly) it in a band of high concentration. At the base of the flow, the concentration field $C_{\infty}(x, 0)$ initially diminishes with distance downstream before reaching a plateau and then increasing again as those particles initially suspended in the upper portion of the flow settle through to the boundary.

For all flows we may evaluate the maximum value of $C_{\infty}$ within the suspension; it is given by

$$
C_{\max }=\frac{\left(\frac{1}{2}-B\right)^{(1-n) / n}+\epsilon}{\epsilon} .
$$



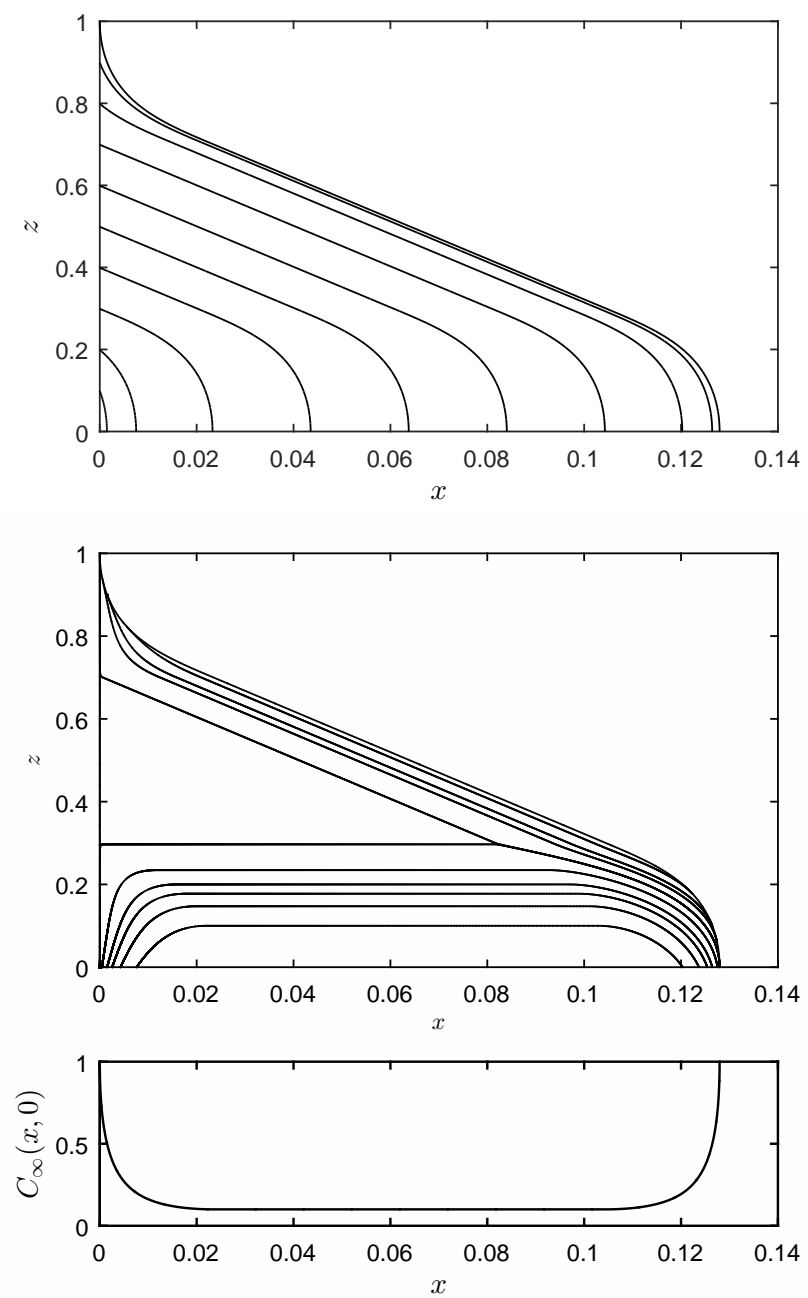

Figure 11: (a) The characteristics in the $(x, z)$ plane; (b) contours of the steady-state concentration field and (c) the steady-state concentration field, $C_{\infty}$, as a function of downstream distance, $x$, at $z=0$ for a sustained release of particles from $x=0$ in a Herschel-Bulkley fluid flowing within a horizontal channel with power-law index $n=1 / 3$, dimensionless yields stress $B=0.2$ and settling parameter $\epsilon=0.01$. Characteristics are plotted for $\xi=0.1-1$ (with interval 0.1 ) and the contours of $C_{\infty}$ are plotted for $C_{\infty}=0.2,0.3,0.4,0.5,0.7,0.999,1.001,1.5,2,4$ and 10. The characteristic from $z=1$ (corresponding to $\xi=1$ ) bounds the region within which the concentration field is non-vanishing. 

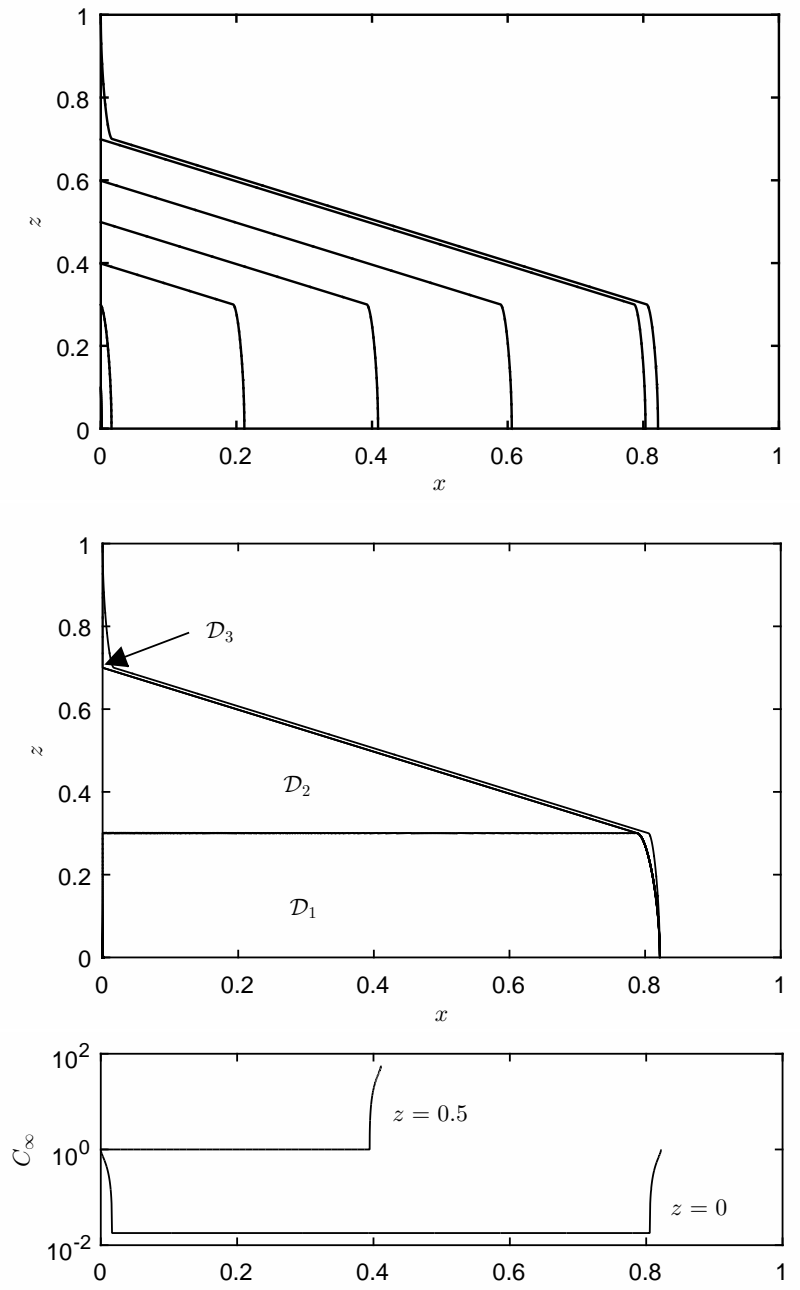

Figure 12: (a) The characteristics in the $(x, z)$ plane; (b) contours of the steady-state concentration field and (c) the steady-state concentration field, $C_{\infty}$, as a function of downstream distance, $x$, at $z=0$ for a sustained release of particles from $x=0$ in a HerschelBulkley fluid flowing within a horizontal channel with power-law index $n=2 / 3$, dimensionless yields stress $B=0.2$ and settling parameter $\epsilon=0.01$. Characteristics are plotted for $\xi=0.3,0.5,0.6,0.7,1.0$ and the contours of $C_{\infty}$ are plotted for $C_{\infty}=0.999,1.001$. The characteristic from $z=1$ (corresponding to $\xi=1$ ) bounds the region within which the concentration field is non-vanishing. Also depicted are the domains $\mathcal{D}_{1}, \mathcal{D}_{2}$ and $\mathcal{D}_{3}$, respectively corresponding to the regions within which $C_{\infty}<0.99,0.99<C_{\infty}<1.01$ and $1.01<C_{\infty}$. 


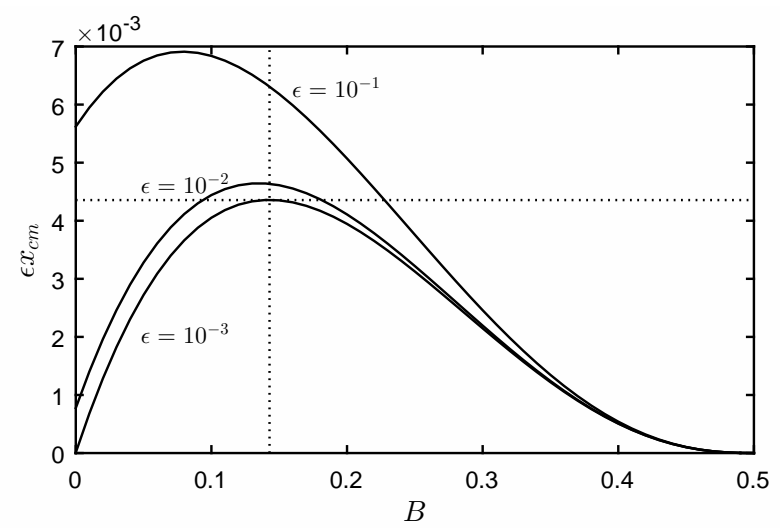

Figure 13: The scaled median distance within the deposit, $\epsilon x_{y m}$, as a function of the dimensionless yield stress, $B$, for a sustained release of particles from $x=0$ in a Herschel-Bulkley fluid flowing within a horizontal channel with power-law index $n=2 / 3$ and settling parameter $\epsilon=0.1,0.01$ and 0.001. Also plotted (dotted lines) is the local maximum $B \equiv B_{m}=n /(4 n+2)$ and $\epsilon x_{y m}=B_{m}\left(\frac{1}{2}-B_{m}\right)^{(n+1) / n}$, attained as $\epsilon \rightarrow 0$

The yield stress has thus led to smaller values of the maximum concentration than found for those suspensions without yield stress ( $c f .(32))$.

Finally we compute the median position within the deposit, here denoted by $x_{y m}$, which is given by the distance downstream at which the characteristic starting from $z=1 / 2$ reaches $z=0$. After some algebraic manipulation, the median position is given by

$$
\begin{gathered}
x_{y m}=\frac{n}{1+n}\left(\int_{0}^{\frac{1}{2}-B} \frac{\left(\frac{1}{2}-B\right)^{(n+1) / n}+\epsilon \eta^{2}}{\eta^{(1-n) / n}+\epsilon} \mathrm{d} \eta-\frac{1}{3}\left(\frac{1}{2}-B\right)^{3}\right. \\
\left.+\frac{B}{\epsilon}\left(\frac{1}{2}-B\right)^{(n+1) / n}\right) .
\end{gathered}
$$

The last term of (45) is due to the settling across the unyielded zone and when $\epsilon \ll 1$, we note that this term provides the leading order expression for the median distance within the deposit. In other words the runout distance of the suspension is controlled entirely by the settling across the unyielded region. Interestingly this median distance varies non-monotonically with $B$ when $\epsilon \ll 1$ reaching a maximum when $B=n /(4 n+2)$ (see figure 13). This non-monotonic variation represents the competition between the slowing of the flow as the yield stress increases, leading to particles being carried less far downstream, and the widening of the kinematic barrier associated with the unyielded region, which leads to increased settling times and an increased distance of transport downstream. 


\section{Conclusions}

In this study, we have constructed a model of the dynamic settling of a suspension of small particles through a sheared flow of a non-Newtonian fluid. In our model, the large-scale flow determines the local effective viscosity, and so the settling speed of the particles varies spatially. This generates a number of phenomena that would not occur if the suspending fluid were Newtonian. From a vertically uniform sustained source, a non-uniform concentration field develops; this may contain regions in which the concentration gradient is statically unstable or in which the concentration is many times higher than it is at source. A key concept to emerge from our analysis is that of the 'kinematic barrier' to settling which occurs near a velocity maximum where shear rates, and thus settling speeds, are low. The kinematic barrier, which is enhanced in viscoplastic fluids because a plug forms around the velocity maximum, can exert strong controls on the run-out and on the pattern of deposition from the flow.

Most of our calculations have been carried out for a power-law fluid rheology. However, the same phenomena could occur in any shear-thinning generalised Newtonian fluid; all that is needed is that the particles are sufficiently small for the larger lengthscale of the background flow to set the shear rate. We have demonstrated how to calculate the solution for any generalised Newtonian fluid provided the settling velocity is a function of the shear rate of the background flow. While computations of drag forces, and thus the settling velocity, could be performed in principle for any imposed rheology, it would be of considerable interest to have experimental confirmation of these flow processes.

Although this contribution has focussed on fundamental insight rather than applications, we reiterate that dynamic settling has important applications in oil extraction and sedimentology. Here we have tackled the simplest flow problems to reveal the rich variety of effects that could occur, but there remain many interesting further problems. These include more concentrated suspensions in which the concentration affects both the settling speed and the density of the flow, and the onset of gravitational overturning as the suspension becomes statically unstable.

\section{Appendix A.}

In this appendix we extend our theoretical developments so that the concentration field may be evaluated in a fully developed, free surface flow of a generalised Newtionian fluid. In terms of dimensional variables and denoting the local shear rate by $\dot{\gamma}=\partial u / \partial z$, the governing equation for the motion is

$$
\frac{\mathrm{d}}{\mathrm{d} z}(\mu(\dot{\gamma}) \dot{\gamma})=-G,
$$

where $\mu(\dot{\gamma})$ is the local viscosity of the generalised Newtonian fluid. The boundary conditions are $\mu(\dot{\gamma}) \dot{\gamma}=0$ on $z=h$ and $u(0)=0$ and thus the solution for 
the velocity field may be written

$$
u=\frac{1}{G} \int_{\dot{\gamma}}^{\dot{\gamma}_{b}}(\mu(q) q)^{\prime} q \mathrm{~d} q
$$

where $\mu\left(\dot{\gamma}_{b}\right) \dot{\gamma}_{b}=G h$ and the prime denotes differentiation. In $\S 2$, the solution for the concentration field was derived in terms of characteristic variables (see (13) and (16)). Assuming that the settling velocity may be written as a function of the local shear rate, $w_{s} \equiv w_{s}(\dot{\gamma})$, we now change the characteristic variables from $(s, \xi)$ to $\left(\dot{\gamma}, \dot{\gamma}_{0}\right)$, where $\dot{\gamma}_{0}$ is the shear rate at $(x, z)=(0, \xi)$, given by $\mu\left(\dot{\gamma}_{0}\right) \dot{\gamma}_{0}=G(h-\xi)$. To change variables we note that

$$
\frac{\mathrm{d} \dot{\gamma}}{\mathrm{d} s}=\frac{\mathrm{d} \dot{\gamma}}{\mathrm{d} z} \frac{\mathrm{d} z}{\mathrm{~d} s}=\frac{G w_{s}(\dot{\gamma})}{(\mu(\dot{\gamma}) \dot{\gamma})^{\prime}}
$$

In terms of the new characteristic variables

$$
\frac{\mathrm{d} x}{\mathrm{~d} \dot{\gamma}}=\frac{u(\dot{\gamma})(\mu(\dot{\gamma}) \dot{\gamma})^{\prime}}{G w_{s}(\dot{\gamma})} \quad \text { and } \quad \frac{\mathrm{d} z}{\mathrm{~d} \dot{\gamma}}=-\frac{(\mu(\dot{\gamma}) \dot{\gamma})^{\prime}}{G}
$$

It is then straightforward to integrate to find the solution given by

$$
\begin{aligned}
& x\left(\dot{\gamma}, \dot{\gamma}_{0}\right)=\int_{\dot{\gamma}_{0}}^{\dot{\gamma}} \int_{q}^{\dot{\gamma}} \frac{(\mu(q) q)^{\prime}(\mu(p) p)^{\prime} p}{G^{2} w_{s}(q)} \mathrm{d} p \mathrm{~d} q \\
& z\left(\dot{\gamma}, \dot{\gamma}_{0}\right)=-\frac{1}{G} \int_{\dot{\gamma}_{0}}^{\dot{\gamma}}(\mu(q) q)^{\prime} \mathrm{d} q=\frac{\mu\left(\dot{\gamma}_{0}\right) \dot{\gamma}_{0}-\mu(\dot{\gamma}) \dot{\gamma}}{G} \\
& s\left(\dot{\gamma}, \dot{\gamma}_{0}\right)=\frac{1}{G} \int_{\dot{\gamma}_{0}}^{\dot{\gamma}} \frac{(\mu(q) q)^{\prime}}{w_{s}(q)} \mathrm{d} q .
\end{aligned}
$$

The solution for the concentration field is written as

$$
C=\mathcal{H}(t-s) \frac{w_{s}\left(\dot{\gamma}_{0}\right)}{w_{s}(\dot{\gamma})}
$$

Acknowledgments: LHC acknowledges the financial support of EPSRC (UK) for funding throughout her Ph.D. studies. DP acknowledges the hospitality of the African Institute of Mathematical Sciences (Ghana) during the preparation of this manuscript. All authors also acknowledge the preliminary calculations on this problem carried out as part of the 20th ECMI Student Modelling Week (2006) by a team comprising Serbiniyaz Anyeva, Liya Asner, Gatis Barauskis, Kho Sinatra Canggih, Miguel Angel Vicente Oliva, Angela Schubert, and Lars Sydnes.

[1] A M Talmon and M Huisman. Fall velocity of particles in shear flow of drilling fluids. Tunnelling and Underground Space Technology, 20:193-201, 2005 .

[2] P Valkó and M J Economides. Hydraulic Fracture Mechanics. Wiley, 1995. 
[3] N P Brown and N I Heywood. Slurry Handling: design of solid-liquid systems. Elsevier Applied Science, 1991.

[4] F Rosso. Complex Flows in Industrial Processes (Ed A. Fasano), chapter Sedimentation in coal-water-slurry pipelining, pages 25-61. Birkäuser, 2000 .

[5] M A Naylor. The origin of inverse grading in muddy debris flow deposits - a review. Journal of Sedimentary Petrology, 50:1111-1116, 1980.

[6] C Ancey. Debris flows and related phenomena. In N J Balmforth and A Provenzale, editors, Geomorphological Fluid Mechanics (Lecture Notes in Physics), page 589. Springer, 2001.

[7] C Lareo, P J Fryer, and M Barigou. The fluid mechanics of two-phase solid-liquid food flows: a review. Trans. I. Chem.E., 75(73-105), 1997.

[8] G Dazhi and R Tanner. The drag on a sphere in a power-law fluid. $J$. Non-Newtonian Fluid Mech., 17:1-12, 1985.

[9] B J Briscoe, P F Luckham, and S R Ren. The settling of spheres in clay suspensions. Powder Technology, 76:165-174, 1993.

[10] G Gheissary and B H A A van den Brule. Unexpected phenomena observed in particle settling in non-Newtonian media. Journal of Non-Newtonian Fluid Mechanics, 67:1-18, 1996.

[11] A N Beris, J A Tsamopoulos, R C Armstrong, and R A Brown. Creeping flow of a sphere through a Bingham plastic. J. Fluid Mech., 158:219-244, 1985 .

[12] E J Novotny. Proppant transport. Society of Petroleum Engineers Paper SPE 6813, presented at 52nd Annual Fall Technical Conference and Exhibition of the Society of Petroleum Engineers, October 197\%., 1977. doi: 10.2118/6813-MS.

[13] L P Roodhart. Proppant settling in non-Newtonian fracturing fluids. Society of Petroleum Engineers / US Department of Energy Paper SPE/DOE 13905, presented at SPE/DOE Low Permeability Gas Reservoirs, May 1985., 1985. doi: 10.2118/13905-MS.

[14] M Asadi, A N Shah, and D L Lord. Static/dynamic settling of proppant in non-Newtonian hydraulic fracturing fluids. Society of Petroleum Engineers Paper SPE 52217, presented at SPE Mid-Continent Operations Symposium, March 1999., 1999. doi: 10.2118/52217-MS.

[15] Y T Hu, H Chung, and J Maxey. What is more important for proppant transport, viscosity or elasticity? Society of Petroleum Engineers Paper SPE 173339-MS, presented at SPE Hydraulic Fracturing Technology Conference, February 2015., 2015. doi: 10.2118/173339-MS. 
[16] P D Scott, M Zamora, and C Aldea. Barite-sag management: challenges, strategies, opportunities. International Association of Drilling Contractors / Society of Petroleum Engineers Paper IADC/SPE 87136, presented at IADC/SPE Drilling Conference, March 2004., 2004. doi: 10.2118/87136MS.

[17] O Merkak, L Jossic, and A Magnin. Dynamics of particles suspended in a yield stress fluid flowing in a pipe. AIChE Journal, 54:1129-1138, 2008. doi: $10.1002 /$ aic.11454.

[18] O Merkak, L Jossic, and A Magnin. Migration and sedimentation of spherical particles in a yield stress fluid flowing in a horizontal cylindrical pipe. AIChE Journal, 55:2515-2525, 2009. doi: 10.1002/aic.11852.

[19] G Ovarlez, F Bertrand, P Coussot, and X Chateau. Shear-induced sedimentation in yield stress fluids. J. Non-Newtonian Fluid Mech., 177-178: 19-28, 2012. doi:10.1016/j.jnnfm.2012.03.013.

[20] E De Angelis and A Mancini. Amodel for the evolution of sedimentation beds in the dynamic of a pipelined non-Newtonian fluid. Mathl. Comput. Modeling, 25:65-78, 1997.

[21] L H Childs. Low Reynolds number flows of generalised non-Newtonian fluids. PhD thesis, School of Mathematics, University of Bristol, U.K., 2013.

[22] M Abramowitz and I Stegun. A handbook of mathematical functions. AMS55 National Bureau of Standards, 1964. 